\title{
Multi-wavelength observations of PKS 2155-304 with HESS
}

\author{
F. Aharonian ${ }^{1}$, A. G. Akhperjanian ${ }^{2}$, A. R. Bazer-Bachi ${ }^{3}$, M. Beilicke ${ }^{4}$, W. Benbow ${ }^{1}$, D. Berge ${ }^{1}$, K. Bernlöhr ${ }^{1,5}$,
} C. Boisson ${ }^{6}$, O. Bolz ${ }^{1}$, V. Borrel ${ }^{3}$, I. Braun ${ }^{1}$, F. Breitling ${ }^{5}$, A. M. Brown ${ }^{7}$, P. M. Chadwick ${ }^{7}$, L.-M. Chounet ${ }^{8}$, R. Cornils ${ }^{4}$, L. Costamante ${ }^{1,20}$, B. Degrange ${ }^{8}$, H. J. Dickinson ${ }^{7}$, A. Djannati-Ataï ${ }^{9}$, L. O'C. Drury ${ }^{10}$, G. Dubus ${ }^{8}$, D. Emmanoulopoulos ${ }^{11}$, P. Espigat ${ }^{9}$, F. Feinstein ${ }^{12}$, G. Fontaine ${ }^{8}$, Y. Fuchs ${ }^{13}$, S. Funk ${ }^{1}$, Y. A. Gallant ${ }^{12}$, B. Giebels ${ }^{8}$, S. Gillessen ${ }^{1}$, J. F. Glicenstein ${ }^{14}$, P. Goret ${ }^{14}$, C. Hadjichristidis ${ }^{7}$, M. Hauser ${ }^{11}$, G. Heinzelmann ${ }^{4}$, G. Henri' ${ }^{13}$, G. Hermann ${ }^{1}$, J. A. Hinton ${ }^{1}$, W. Hofmann ${ }^{1}$, M. Holleran ${ }^{15}$, D. Horns ${ }^{1}$, A. Jacholkowska ${ }^{12}$, O. C. de Jager ${ }^{15}$, B. Khélifi ${ }^{1}$, Nu. Komin ${ }^{5}$, A. Konopelko ${ }^{1,5}$, I. J. Latham ${ }^{7}$, R. Le Gallou ${ }^{7}$, A. Lemière ${ }^{9}$, M. Lemoine-Goumard ${ }^{8}$, N. Leroy ${ }^{8}$, T. Lohse ${ }^{5}$, J. M. Martin ${ }^{6}$, O. Martineau-Huynh ${ }^{16}$, A. Marcowith ${ }^{3}$, C. Masterson ${ }^{1,20}$, T. J. L. McComb ${ }^{7}$, M. de Naurois ${ }^{16}$, S. J. Nolan ${ }^{7}$, A. Noutsos ${ }^{7}$, K. J. Orford ${ }^{7}$, J. L. Osborne ${ }^{7}$, M. Ouchrif ${ }^{16,20}$, M. Panter ${ }^{1}$, G. Pelletier ${ }^{13}$, S. Pita ${ }^{9}$, G. Pühlhofer ${ }^{1,11}$, M. Punch ${ }^{9}$, B. C. Raubenheimer ${ }^{15}$, M. Raue ${ }^{4}$, J. Raux ${ }^{16}$, S. M. Rayner ${ }^{7}$, A. Reimer ${ }^{17}$, O. Reimer ${ }^{17}$, J. Ripken ${ }^{4}$, L. Rob ${ }^{18}$, L. Rolland ${ }^{16}$, G. Rowell ${ }^{1}$, V. Sahakian ${ }^{2}$, L. Saugé ${ }^{13}$, S. Schlenker ${ }^{5}$, R. Schlickeiser ${ }^{17}$, C. Schuster ${ }^{17}$, U. Schwanke ${ }^{5}$, M. Siewert ${ }^{17}$, H. Sol ${ }^{6}$, D. Spangler ${ }^{7}$, R. Steenkamp ${ }^{19}$, C. Stegmann ${ }^{5}$, J.-P. Tavernet ${ }^{16}$, R. Terrier ${ }^{9}$, C. G. Théoret ${ }^{9}$, M. Tluczykont ${ }^{8,20}$, G. Vasileiadis $^{12}$, C. Venter ${ }^{15}$, P. Vincent ${ }^{16}$, H. J . Völk ${ }^{1}$, and S. J. Wagner ${ }^{11}$

\begin{abstract}
${ }^{1}$ Max-Planck-Institut für Kernphysik, Heidelberg, Germany; ${ }^{2}$ Yerevan Physics Institute, Armenia; ${ }^{3}$ Centre d'Étude Spatiale des Rayonnements, CNRS/UPS, Toulouse, France; ${ }^{4}$ Universität Hamburg, Institut für Experimentalphysik, Germany; ${ }^{5}$ Institut für Physik, Humboldt-Universität zu Berlin, Germany; ${ }^{6}$ LUTH, UMR 8102 du CNRS, Observatoire de Paris, Section de Meudon, France;

${ }^{7}$ University of Durham, Department of Physics, UK; ${ }^{8}$ Laboratoire Leprince-Ringuet, IN2P3/CNRS, École Polytechnique, Palaiseau, France, e-mail: berrie@poly.in2p3.fr; 9 APC, Paris, France ${ }^{\star} ;{ }^{10}$ Dublin Institute for Advanced Studies, Ireland; ${ }^{11}$ Landessternwarte, Königstuhl, Heidelberg, Germany; ${ }^{12}$ Laboratoire de Physique Théorique et Astroparticules, IN2P3/CNRS, Université Montpellier II, France; ${ }^{13}$ Laboratoire d'Astrophysique de Grenoble, INSU/CNRS, Université Joseph Fourier, France; ${ }^{14}$ DAPNIA/DSM/CEA, CE Saclay, Gif-sur-Yvette, France; ${ }^{15}$ Unit for Space Physics, North-West University, Potchefstroom, South Africa; ${ }^{16}$ Laboratoire de Physique Nucléaire et de Hautes Énergies, IN2P3/CNRS, Universités Paris VI \& VII, Paris, France; ${ }^{17}$ Institut für Theoretische Physik, Lehrstuhl IV: Weltraum und Astrophysik, Ruhr-Universität Bochum, Germany; ${ }^{18}$ Institute of Particle and Nuclear Physics, Charles University, Prague, Czech Republic; ${ }^{19}$ University of Namibia1, Windhoek, Namibia; ${ }^{20}$ European Associated Laboratory for Gamma-Ray Astronomy, jointly supported by CNRS and MPG
\end{abstract}

Received 2 May 2005 / Accepted 22 June 2005

\section{ABSTRACT}

The High Energy Stereoscopic System (HESS) has observed the high-frequency peaked BL Lac object PKS $2155-304$ in 2003 between October 19 and November 26 in Very High Energy (VHE) $\gamma$-rays ( $E \geq 160 \mathrm{GeV}$ for these observations). Observations were carried out simultaneously with the Proportional Counter Array (PCA) on board the Rossi X-ray Timing Explorer satellite (RXTE), the Robotic Optical Transient Search Experiment (ROTSE) and the Nançay decimetric radiotelescope (NRT). Intra-night variability is seen in the VHE band, the source being detected with a high significance on each night it was observed. Variability is also found in the X-ray and optical bands on kilosecond timescales, along with flux-dependent spectral changes in the X-rays. A transient X-ray event with a $1500 \mathrm{~s}$ timescale is detected, making this the fastest X-ray flare seen in this object. No correlation can be established between the X-ray and the $\gamma$-ray fluxes, or any of the other wavebands, over the small range of observed variability. The average HESS spectrum shows a very soft power law shape with a photon index of $3.37 \pm 0.07_{\text {stat }} \pm 0.10_{\text {sys }}$. The energy outputs in the $2-10 \mathrm{keV}$ and in the VHE $\gamma$-ray range are found to be similar, with the X-rays and the optical fluxes at a level comparable to some of the lowest historical measurements, indicating that PKS 2155-304 was in a low or quiescent state during the observations. Both a leptonic and a hadronic model are used to derive source parameters from these observations. These parameters are found to be sensitive to the model of Extragalactic Background Light (EBL) that attenuates the VHE signal at this source's redshift $(z=0.117)$.

Key words. galaxies: active - gamma rays: observations - X-rays: galaxies - radiation mechanisms: non thermal

^ UMR 7164 (CNRS, Université Paris VII, CEA, Observatoire de Paris). 


\section{Introduction}

The innermost regions of active galactic nuclei, where the largest part of their luminosity is emitted, can be probed through observations of their flux variability at different wavelengths. The physical processes in their central engines and jets are usually considered the main candidates for the origin of the observed variability. Measurements of correlated variability, spectral variations and time lags across the broad-band observations allow modelling of particle distributions and their radiation processes, as well as probing the acceleration mechanisms that are involved.

PKS 2155-304 is probably the most prominent and beststudied blazar-type Active Galactic Nuclei (AGN) in the Southern Hemisphere. The emission of PKS 2155-304, its possible variability patterns, as well as correlations across all wavebands, have been studied exhaustively over the past 20 years (see e.g. Urry et al. 1997). Its first detection at VHE $\gamma$-rays by the Durham Mk VI telescopes (Chadwick et al. 1999) classified it as a TeV blazar, like the northern hemisphere BL Lac objects Mkn 421, Mkn 501, $\mathrm{H} 1426+428$, or $1 \mathrm{ES} 1959+650$. Its redshift of $z=0.117$ makes it the second most distant confirmed TeV blazar after $\mathrm{H} 1426+428(z=0.129)$. PKS 2155-304 was the brightest BL Lac object in the EUVE all-sky survey (Marshall et al. 1995). This source was confirmed as a high energy $\gamma$-ray emitter by HESS (Aharonian et al. 2005, AH04 hereafter) at the $45 \sigma$ significance level, when strong detections were reported for each of the dark periods of observations.

Here we report on simultaneous HESS VHE $\gamma$-ray, RXTE/PCA X-ray, ROTSE optical, and NRT decimetric observations of PKS 2155-304 during the dark periods of October and November 2003. No simultaneous multiwavelength campaign had before included an Atmospheric Cherenkov Telescope (ACT) that could sample the evolution of the high energy component of the spectral energy distribution (SED) of this object. We also include EGRET archival data, and other archival radio through X-ray data obtained from the NASA/IPAC Extragalactic Database (NED). Details of the observations and data reduction/analysis are given in Sect. 2. Light curves and spectra are described in Sect. 3. The attenuation of the HESS spectrum by the EBL and an interpretation of the data using a leptonic and a hadronic model are discussed in Sect. 4.

\section{Observations and data analysis}

\subsection{HESS}

\subsubsection{HESS detector}

In its first phase, the HESS array consists of four atmospheric Cherenkov telescopes operating in stereoscopic mode. However, the data shown here were taken during the construction of the system, initially with two telescopes, with one more as of mid-September, 2003. The fourth and final telescope was added to the array in December 2003, subsequent to the data presented here. Each telescope has a tessellated $13 \mathrm{~m}$-diameter (107 $\mathrm{m}^{2}$ surface area) mirror which focuses the Cherenkov light from the showers of secondary particles created by the interaction of $\gamma$-rays in the atmosphere onto a camera in the focal plane. This camera consists of 960 photomultipliers, each with a pixel size of $0.16^{\circ}$, giving a field of view of $5^{\circ}$. For the data sets presented here the $\gamma$-ray trigger threshold is $\approx 100 \mathrm{GeV}$ and the spectral threshold is $160 \mathrm{GeV}$ with an energy resolution $\simeq 15 \%$. The experiment is located in the Khomas highlands in Namibia, $\left(23^{\circ} \mathrm{S}, 15^{\circ} \mathrm{E}, 1800 \mathrm{~m}\right.$ a.s.1.). A detailed description of the layout and the components of the telescope optical systems, including the segmented mirror with its support structure, the mirror facets for each telescope and the Winston cone light concentrators in front of the PMT camera can be found in Bernlöhr et al. (2003), and a description of the mirror alignment is given in Cornils et al. (2003). For details on the camera calibration see Aharonian et al. (2004a). The trigger system is described in Funk et al. (2004). Early reports of HESS have been given elsewhere (see e.g. Hofmann 2002).

\subsubsection{Observations}

In the beginning of the observation period of October 2003, a single $5 \sigma$ detection by HESS achieved in $1 \mathrm{~h}$, triggered an approved RXTE ToO (Target of Opportunity) on this target. The results presented here are based on observations carried out in 2003 between October 19 and November 26.

The data were taken in the Wobble mode where the source direction is positioned $\pm 0.5^{\circ}$ in declination relative to the centre of the field of view of the camera during observations. This allows for both on-source observations and simultaneous estimation of the background induced by charged cosmic rays. The data reported here are selected and analyzed with the "standard analysis" described in Sect. 4 of AH04. The background is estimated here by using all events passing cuts in a ring around the source, as described in Sect. 4.3 in AH04. The runs passing the quality selection criteria total $32.4 \mathrm{~h}$ of livetime on the source. The total two-dimensional significance sky map is shown in Fig. 1, along with a graph showing the $\theta^{2}$ distribution (where $\theta^{2}$ is the square of the angular difference between the reconstructed shower position and the source position) of the 1764 excess events observed. This yields a detection at the $34.3 \sigma$ level, at the average rate of $0.91 \gamma / \mathrm{min}$ and a significance of $6.0 \sigma / \sqrt{h}$.

The methods used here for reconstructing the energy of each event and for determining a spectrum are described in Sect. 6 of AH04. The measured time-average spectrum is fitted by a power law of the form $\mathrm{d} N / \mathrm{d} E=I_{0}(E / 1 \mathrm{TeV})^{-\Gamma}$ with $I_{0}$ the flux normalization at $1 \mathrm{TeV}$ and $\Gamma$ the photon index. The photon index obtained from the time-averaged spectrum is then used as a fixed parameter to estimate the integral flux above $300 \mathrm{GeV}$ for each run. This integrated flux takes into account the effective area and threshold variations due to the source moving through the sky, giving more reliable variability information than counting rates in units of $\gamma$-rays/min. Overall systematic errors are estimated to be $20 \%$ for the integral flux and $\simeq 0.1$ for the photon index. 

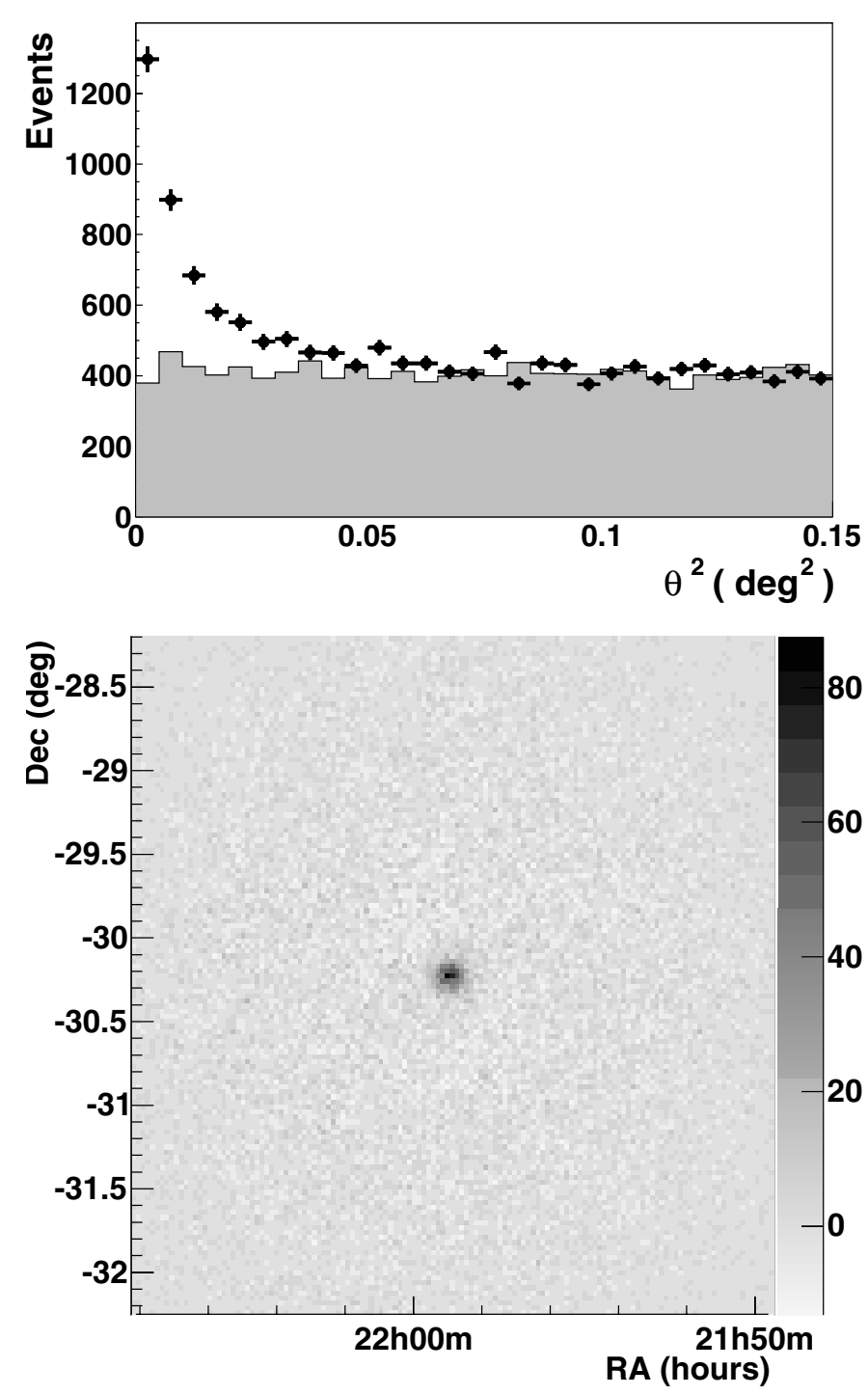

Fig. 1. Top: the distribution of $\theta^{2}$ for on-source events (points) and normalized off-source events (shaded) for the observations in October and November 2003. Bottom: the two-dimensional distribution of excess events observed in the direction of PKS 2155-304. The bins are not correlated and represent the actual distribution of observed $\gamma$-rays on the sky. The right hand scale is the number of counts.

\subsection{RXTE}

The PCA (Jahoda et al. 1996) units of RXTE observed PKS 2155-304 between October 22 and November 23 of 2003 with exposures of typically $10 \mathrm{ks}$ in October and $\simeq 1 \mathrm{ks}$ in November. The STANDARD2 data were extracted using the ftools in the HEASOFT 5.3.1 analysis software package provided by NASA/GSFC and filtered using the RXTE Guest Observer Facility (GOF) recommended criteria. The changing Proportional Counter Units (PCU0/2/3) configuration throughout the observations was taken into account in the data reduction, and only the signals from the top layer (X1L and X1R) were used. When reducing the PCUs individually to establish the time-averaged spectrum, the average spectral fit parameters are similar within error bars but with a systematically higher $\chi^{2}$ for spectral fits performed on PCU0 data alone.
On shorter timescales this effect becomes negligible and PCU0 contributes to the statistical significance of the flux measurement. Therefore all PCUs were kept for the overall light curve which is binned in $400 \mathrm{~s}$ bins, but only PCU2 and PCU3 are used for the analysis of data segments that are simultaneous with HESS runs and for the time-averaged spectrum.

The average spectrum used in the SED is derived by combining PCU2 and PCU3 spectra using the addspec tool weighted by the counts information delivered by fstatistic and then the corresponding response matrices were combined with addrmf. The faint-background model was used and only the 3-40 PHA channel range was kept in XSPEC v . 11.3.1, or approximately $2-20 \mathrm{keV}$.

To build a light curve in units of integrated flux in the $2-10 \mathrm{keV}$ band, spectral data were derived from $400 \mathrm{~s}$ bins to probe short timescales with adequate statistical accuracy. These segments are then fitted by a power law in XSPEC with PCU configuration-dependent response matrices generated by the ftool pcarsp v. 10.1 and a fixed column density of $N_{\mathrm{H}}=1.7 \times 10^{20} \mathrm{~cm}^{-2}$ obtained from PIMMS ${ }^{1}$. This yields the flux and the error (corresponding to the $1 \sigma$ confidence level) on the flux reported in the light curves in Fig. 2, in units of $10^{-11} \mathrm{erg} \mathrm{cm}^{-2} \mathrm{~s}^{-1}$ in the $2-10 \mathrm{keV}$ band. The fits did not improve by using a broken power-law for the $400 \mathrm{~s}$ binned observations.

\subsection{ROTSE}

The ROTSE-III array is a worldwide network of four $0.45 \mathrm{~m}$ robotic, automated telescopes built for fast $(\approx 6 \mathrm{~s})$ response to GRB triggers from satellites such as HETE-2 (High Energy Transient Explorer 2) and Swift. The ROTSE-III telescopes have a wide $\left(1.85^{\circ} \times 1.85^{\circ}\right)$ field of view imaged onto a Marconi $2048 \times 2048$ pixel back-illuminated thinned CCD and are operated without filters. The ROTSE-III systems are described in detail in Akerlof et al. (2003). At the time of the observations of PKS 2155-304 in October and November 2003, two ROTSE-III telescopes were operational in the Southern hemisphere: ROTSE-IIIa located at the Siding Spring Observatory, Australia and ROTSE-IIIc at the HESS site. The ROTSE-IIIc telescope is located in the centre of the HESS telescope array. A $30 \%$ share of the total observation time is available to the HESS collaboration, which has been used to perform an automated monitoring programme of blazars, including objects that are being observed with the HESS telescopes. Both telescopes participated in the observation campaign on PKS 2155-304 in October and November 2003.

The telescopes observed PKS 2155-304 typically 10 times per night taking sequences of 2 frames with 60 s exposures with a slight dithering of the pointing to reduce the impact of individual noisy pixels. The typical limiting magnitude, depending on the sky conditions, is $18.5^{\mathrm{mag}}$. Overall, 323 bias-subtracted and flat-fielded frames have passed visual inspection and are used to produce a light curve. A total of 6 frames were rejected due to the presence of stray light from Jupiter.

\footnotetext{
${ }^{1}$ See http://legacy.gsfc.nasa.gov/Tools/w3pimms.html
} 

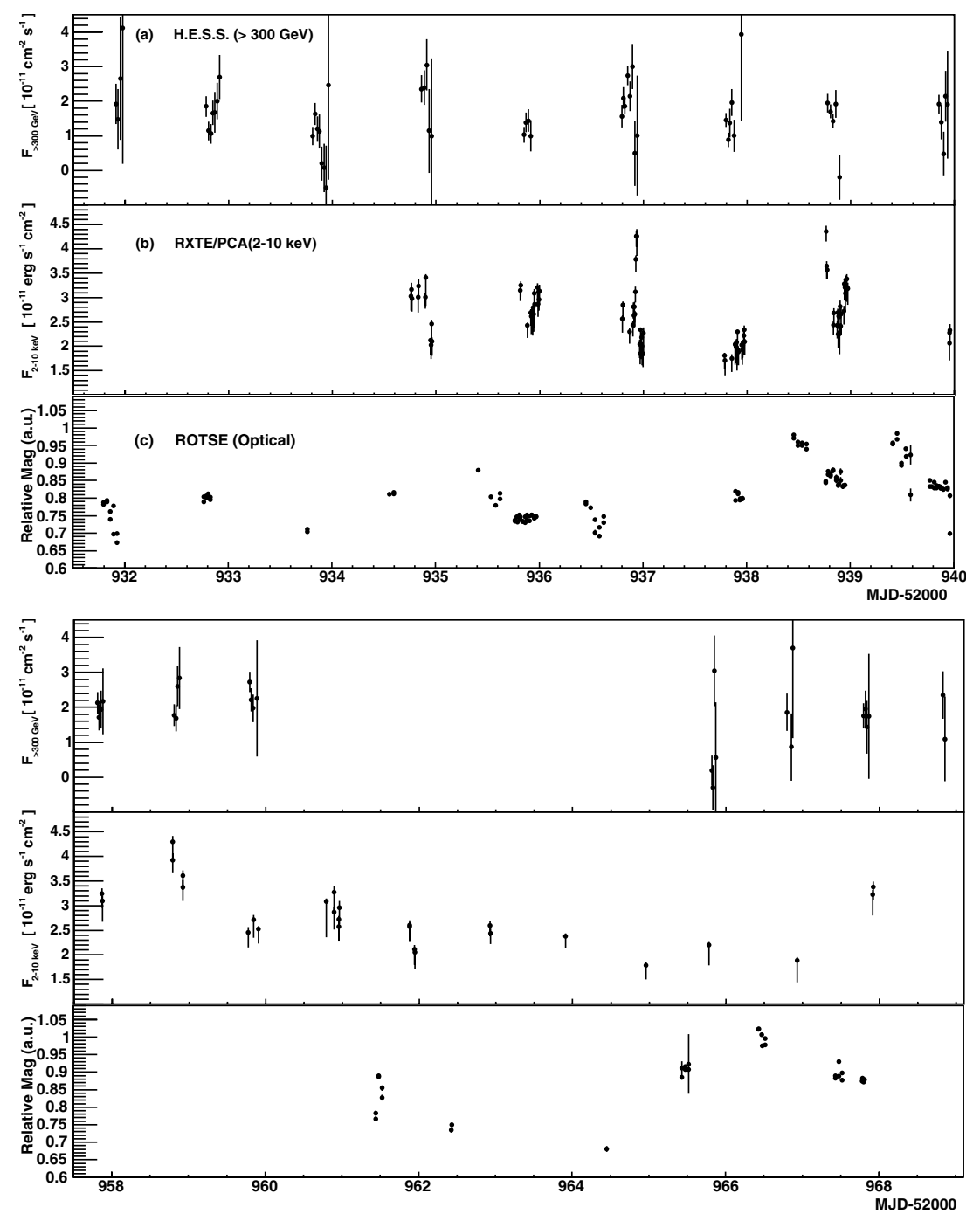

Fig. 2. Top: a) October 2003 Light curve from HESS binned in run lengths averaging 28 min each, in units of integral flux above $300 \mathrm{GeV}$; b) light curve expressed as flux in the $2-10 \mathrm{keV}$ band for the RXTE observations, in $400 \mathrm{~s}$ bins; c) light curve derived from the ROTSE optical data. Bottom: same as above but for the November 2003 data. Note that the RXTE observations were much shorter than in the previous month.

Using an overlay of 50 isolated comparison stars with similar brightness $\left(12^{\mathrm{mag}}-14^{\mathrm{mag}}\right)$ and co-located with PKS 2155-304 (<15 arcmin), a two-dimensional Gaussian is fit to the intensity distribution characterising the point-spread function by $\sigma_{\mathrm{psf}}$.

To estimate the local sky-background for the reference stars and the target object, an annulus with inner radius $2 \times \sigma_{\mathrm{psf}}$ and an outer radius $6 \times \sigma_{\mathrm{psf}}$ is chosen. Based upon a reference frame which is derived from co-adding 30 individual frames, a mask is calculated for each object excluding regions where faint objects coincide with the annulus; pixels exceeding 3 standard deviations of the local sky background are excluded. Using the local sky background, the intensity and error of each object is calculated. Using the 50 reference stars, a relative intensity and statistical error with respect to a reference frame is calculated.

The absolute flux values are obtained by calculating a relative $R$ magnitude by comparing the instrumental magnitude with the USNO catalogue as described in Akerlof et al. (2000). The procedure has been checked by comparing the average $R$ magnitude of a sample of $70 \mathrm{BL}$ Lac type objects determined with ROTSE observations carried out over one year of operation with the $V$ magnitude listed in the 10th Veron Cetty \& Veron catalogue of BL Lac type objects. The average $V-R$ of 0.5 that is found is consistent with the average value for $V-R$ obtained from cross-checking the colours with the 2MASS catalogued value for the BL Lac type objects.

Finally, the host galaxy has been resolved in optical (Falomo 1996) and NIR (Kotilainen et al. 1998, KFS98 hereafter) and found to be a typical giant elliptical with $M(R)=$ -24.4 which translates into an apparent $m(R)=15.1$ (here the distance moduli given by KFS98 have been used to calculate the apparent magnitude based upon the absolute magnitude quoted). The ROTSE measurements have as maximum and minimum $m(R, \min )=13.3$ and $m(R, \max )=13.7$ which corresponds to $10 \mathrm{mJy}$ for the maximum observed flux and $6.7 \mathrm{mJy}$ for the minimum flux taking the contribution of the host galaxy into account. These values are considerably lower than the retrieved archival data indicating that PKS 2155-304 was in a low state at the moment of the observations. 


\subsection{NRT}

The Nançay radiotelescope is a single-dish antenna with a collecting area of $200 \times 34.56 \mathrm{~m}^{2}$ equivalent to that of a $94 \mathrm{~m}$ diameter parabolic dish (van Driel et al. 1996). The half-power beam width at $11 \mathrm{~cm}$ is $1.9 \operatorname{arcmin}(E W) \times 11.5 \operatorname{arcmin}(\mathrm{NS})$ (at zero declination), and the system temperature is about $45 \mathrm{~K}$ in both horizontal and vertical polarizations. The point source efficiency is $0.8 \mathrm{~K} \mathrm{Jy}^{-1}$, and the chosen filter bandwidth was 12.5 $\mathrm{MHz}$ for each polarization, split into two sub-bands of 6.25 MHz each. Between 4 and 14 individual 1-min drift scans were performed for each observation, and the flux was calibrated using a calibrated noise diode emission for each drift scan. Data processing has been done with the Nançay local software NAPS and SIR.

A monitoring programme with this telescope on extragalactic sources visible by both the NRT and HESS is in place since 2001. For the campaign described here it consisted of a measurement at $11 \mathrm{~cm}$ every two or three days. The average flux for the 8 measurements in October and November 2003 was $0.30 \pm 0.01 \mathrm{Jy}$ with possible marginal variability.

\section{Results}

\subsection{Light curves}

The October and November 2003 light curves of all the HESS, RXTE and ROTSE observations are shown in Fig. 2. The HESS light curve is binned in run-length times averaging $28 \mathrm{~min}$ each. The flux is in units of $10^{-11}$ photons $\mathrm{cm}^{-2} \mathrm{~s}^{-1}$ above $300 \mathrm{GeV}$, derived using the average photon index 3.37 obtained in Sect. 3.3. Spectra could not be derived on a run-by-run basis due to the weak signal. As for the observations reported in $\mathrm{AH} 04$, the overall light curve is inconsistent with a constant flux. A $\chi^{2}$ fit of the data to a constant yields a $3.4 \times 10^{-10} \chi^{2}$ probablility. The intra-night VHE flux on MJD 52936 (Fig. 3) exhibits an increase of a factor of $1.9 \pm 0.6$ in $0.11 \mathrm{~d}$. On MJD 52932 the peak-to-peak flux shows an increase of a factor of $2.5 \pm 0.9$ within $0.09 \mathrm{~d}$. These timescales are longer than the 30 min doubling time reported in $\mathrm{AH} 04$. For these two extreme cases of VHE variability observed during this campaign only the second had a limited RXTE coverage.

The $2-10 \mathrm{keV} X$-ray flux in this campaign ranges from $F_{2-10 \mathrm{keV}}=2.0 \times 10^{-11} \mathrm{erg} \mathrm{cm}^{-2} \mathrm{~s}^{-1}$ to $4.4 \times 10^{-11} \mathrm{erg} \mathrm{cm}^{-2} \mathrm{~s}^{-1}$. The maximum is lower than the 20 November 1997 measurement of $2.3 \times 10^{-10} \mathrm{erg} \mathrm{cm}^{-2} \mathrm{~s}^{-1}$ (Vestrand \& Sreekumar 1999) indicating that the X-ray state seen here is not exceptionally high. The minimum seen here is consistent with historically low fluxes (Zhang et al. 2002). The intra-night variability is also obvious here, but no flare was completely resolved.

A $60 \%$ flux variability in $t_{\mathrm{var}} \approx 1.5 \mathrm{ks}$ on MJD 52936 is the best marked transient episode in the observations reported here (bottom panel b) in Fig. 3 for which the HESS observations were made at the end of the transit inducing a large associated error on the flux estimation due to the high zenith angle of the source. This timescale is comparable to those reported by Gaidos et al. (1996) where doubling times as short as $15 \mathrm{~min}$ from Mkn 421 were observed in the VHE band.

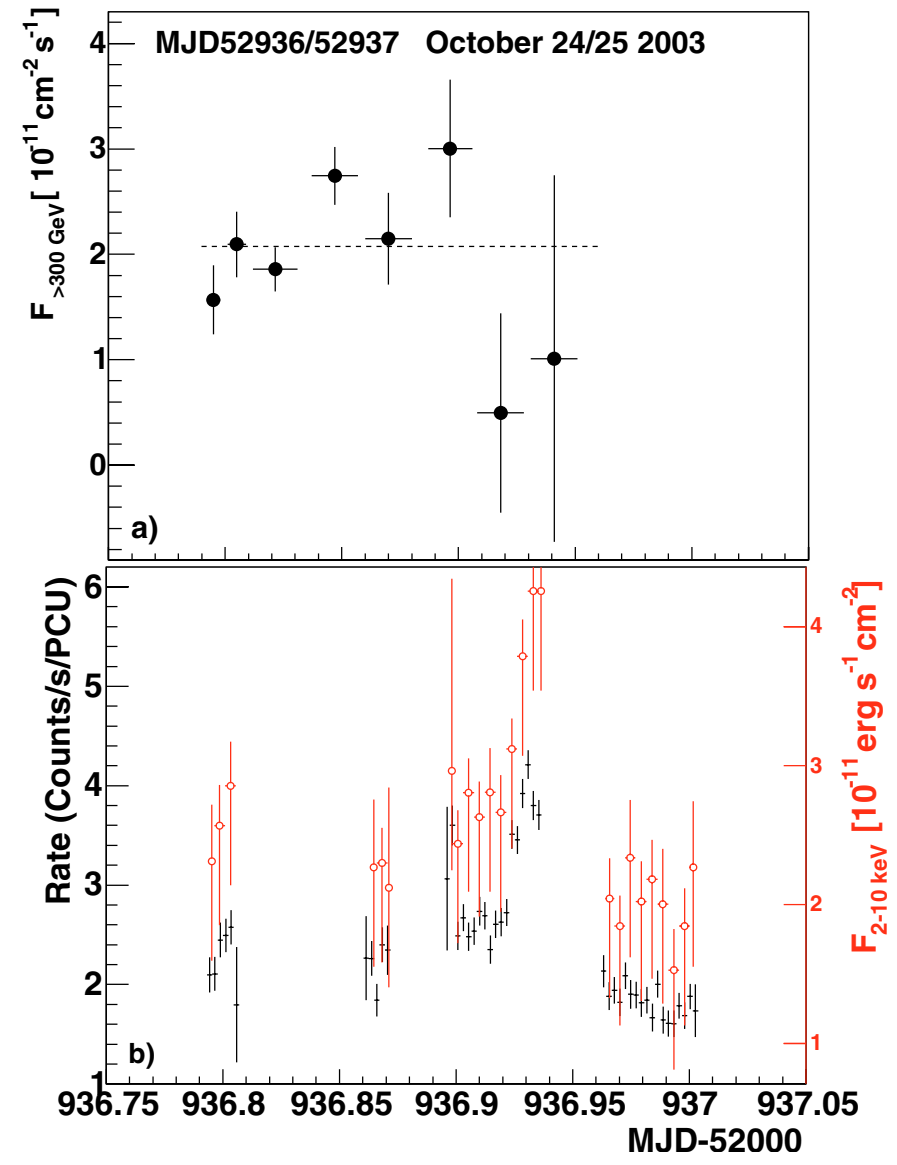

Fig. 3. a) The VHE light curve derived run by run on MJD 52936 . The horizontal error bars are the length of the run from which the flux is derived. The dashed line is the result of a $\chi^{2}$ fit of the data to a constant, which yields a $\chi^{2}$ of 15 for 7 degrees of freedom, corresponding to a $0.04 \chi^{2}$ probability which is evidence for the variability seen here. b) The $2-10 \mathrm{keV} \mathrm{X}$-ray flux (open circles, right scale) and counting rate normalized to $1 \mathrm{PCU}$ (lines, left scale) showing the fast transient.

This flare is the fastest rise seen in this object to date since BeppoSAX saw a $5 \times 10^{4}$ s rise timescale (Zhang et al. 2002) and Kataoka et al. (2000) observed a doubling timescale of $3 \times 10^{4} \mathrm{~s}$ with the ASCA satellite. So far the fastest rise in this type of object was observed in Mkn 501 with a $60 \%$ increase in less than $200 \mathrm{~s}$ (Catanese \& Sambruna 2000) though Xue \& Cui (2004) claims that this flare is likely to be an artifact.

The optical emission of PKS 2155-304 is dominated by the nucleus which outshines the host galaxy by a factor of $\approx 4$ given in KFS98. The observed variability amplitude is therefore not biased by the constant emission of the host galaxy and mainly due to the activity of the nucleus. The peak-to-peak amplitude of variability is moderate compared to the variability amplitude at shorter wavelengths and typically $0.1^{\mathrm{mag}}$ peak-to-peak. The object has been monitored over longer time-scales with the ROTSE-IIIc telescopes showing variations with amplitudes close to $1^{\mathrm{mag}}$. 

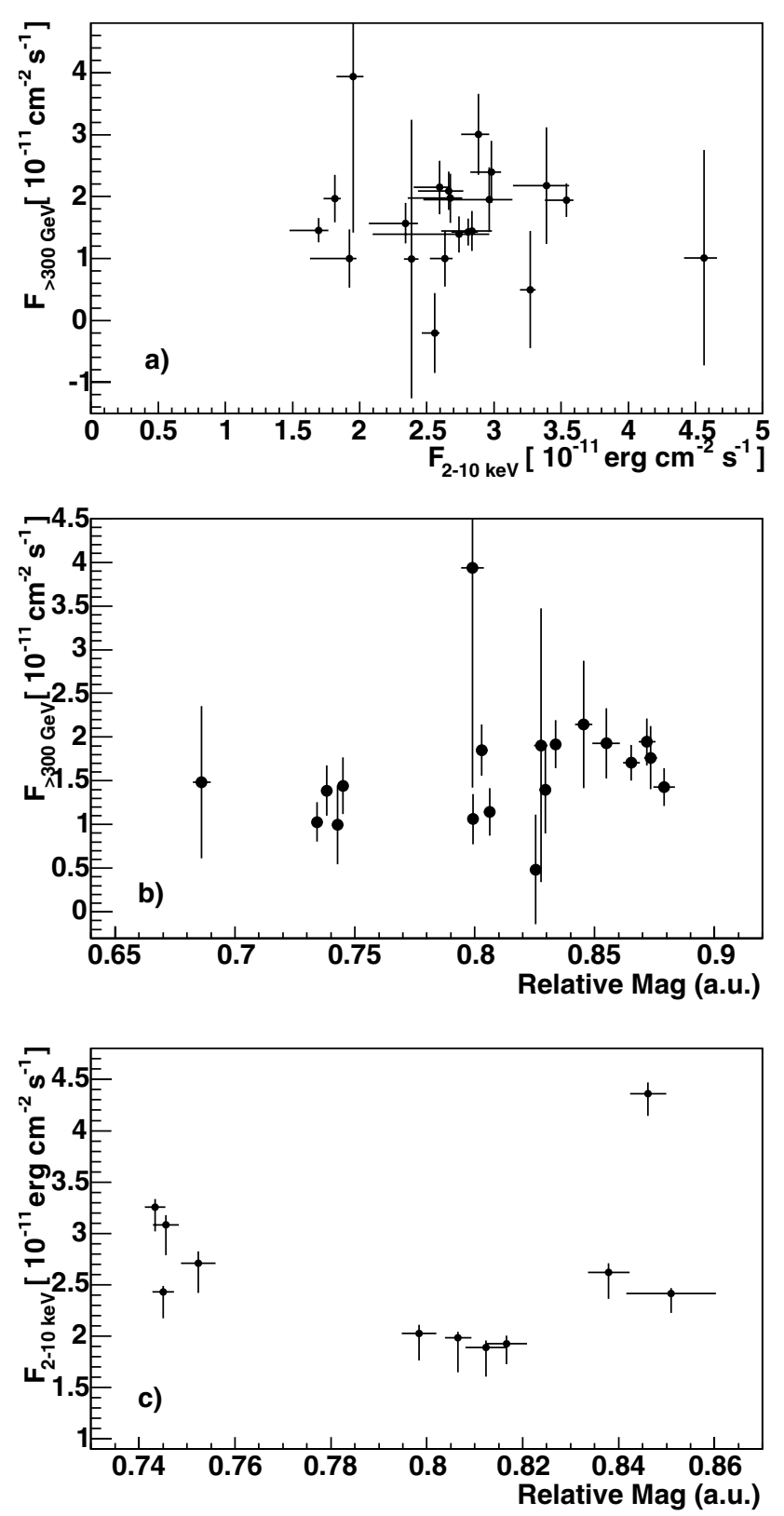

Fig. 4. Correlation plots between different wavebands. No clear correlation is found between any of the simultaneous measurements. a) Correlation plot for the $23 \mathrm{X}$-ray data segments that overlapped exactly with a HESS observation. b) Simultaneous HESS and ROTSE observations. c) Simultaneous RXTE and ROTSE observations. The optical data were binned to the $400 \mathrm{~s}$ long RXTE segments, which are overlapping on 6 different days.

\subsection{Correlation analysis}

In order to quantitatively look for correlated variability between the VHE, X-ray and optical bands, the measured fluxes are plotted against each other in Fig. 4 for all the observations carried out during this campaign. For correlated $V H E / \mathrm{X}$-ray variability, the RXTE analysis was slightly modified: only observation segments that happen exactly within a HESS run are reduced and analyzed (using only PCU2, PCU3 and combinations thereof). This provides 23 simultaneous data segments for the whole campaign for which the fluxes are represented

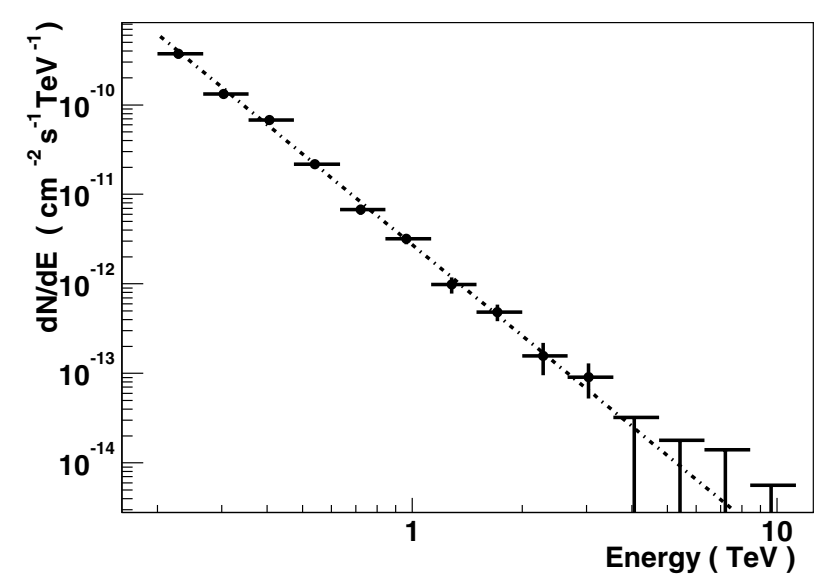

Fig. 5. Time-averaged spectrum derived from the October and November 2003 HESS data along with a fit to a powerlaw.

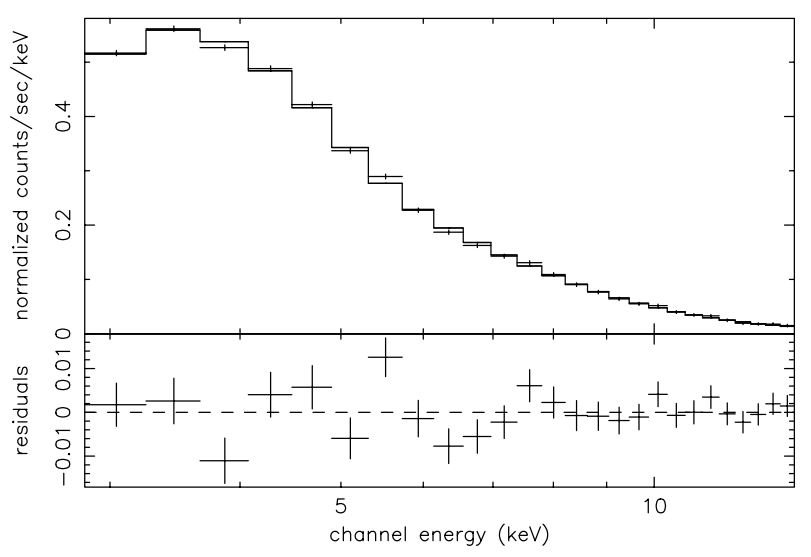

Fig. 6. X-ray spectrum derived from the summed October and November 2003 data, using PCU2 and PCU3, fitted by a broken power law. The top panel shows the data and folded model, the bottom panel shows the residuals between the data and the model.

in Fig. 4. There is no obvious correlation for those observations (correlation factor $r=0.37 \pm 0.13$ ). For correlated opti$\mathrm{cal} /$ VHE variability, ROTSE observations that happen within a HESS run are averaged and their errors summed quadratically. No correlation ( $r=0.24 \pm 0.27)$ is found for these observations. Also no correlation was found between the optical and X-ray band $(r=-0.02 \pm 0.05)$.

\subsection{Spectra}

The October and November 2003 HESS data were all combined for the spectrum shown in Fig. 5. The best-fitting power law ( $\chi^{2}=7$ for 8 degrees of freedom) is given by:

$$
\frac{\mathrm{d} N}{\mathrm{~d} E}=(2.73 \pm 0.17) \times 10^{-12}\left(\frac{E}{\mathrm{TeV}}\right)^{-3.37 \pm 0.07 \pm 0.10}
$$

which is comparable to $\Gamma=3.32 \pm 0.06$ and $I_{0}=(1.96 \pm 0.12) \times$ $10^{-12} \mathrm{~cm}^{-2} \mathrm{~s}^{-1} \mathrm{TeV}^{-1}$ previously reported in AH04.

The result of the broken power law fit for the combined RXTE PCU2 and PCU3 spectrum is shown in Fig. 6. It yields an unabsorbed flux in the $2-10 \mathrm{keV}$ band of 


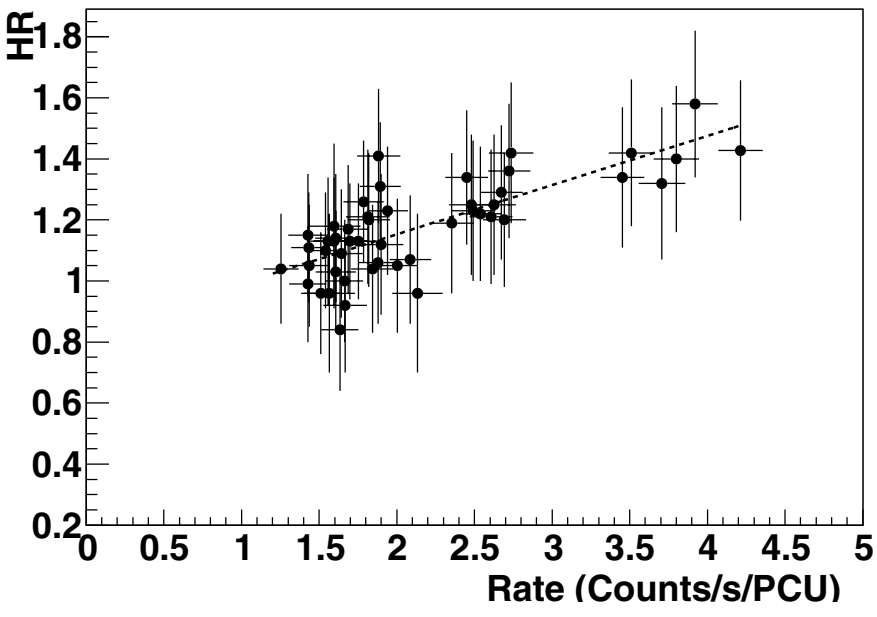

Fig. 7. Plot of the hardness ratio HR versus the counting rate normalized to $1 \mathrm{PCU}$.

$F_{2-10 \mathrm{keV}}=(2.66 \pm 0.04) \times 10^{-11} \mathrm{erg} \mathrm{cm}^{-2} \mathrm{~s}^{-1}\left(\chi^{2}=41\right.$, 31 degrees of freedom), a lower index of $\Gamma_{L}=2.81 \pm 0.05$, a break energy of $E_{\mathrm{b}}=4.9 \pm 0.8 \mathrm{keV}$ and a higher index of $\Gamma_{\mathrm{H}}=2.95 \pm 0.04$. A single power law fit to the same data yields $F_{2-10 \mathrm{keV}}=(2.69 \pm 0.03) \times 10^{-11} \mathrm{erg} \mathrm{cm}^{-2} \mathrm{~s}^{-1}$ $\left(\chi^{2}=51,34\right.$ degrees of freedom $)$ and an index of $\Gamma=$ $2.88 \pm 0.13$, a poorer fit than the broken power law, but the index still provides information that can be used for comparison with historical measurements. Indeed, the derived index is close to those measured by the BeppoSAX satellite (Giommi et al. 1998), GINGA (Sembay et al. 1993) and well within the range observed by EXOSAT (Treves et al. 1989). The statistics above $10 \mathrm{keV}$ in our RXTE observations are too poor to check the existence of a possible hard tail above $20 \mathrm{keV}$ (Giommi et al. 1998) which might be the signature of the onset of a high-energy component.

In order to look for flux dependent spectral variability, the RXTE data subset used in Fig. 3 is divided into two energy bands, the PHA channels 0-9 (soft band) and 10-27 (hard band), corresponding to approximately $1-4 \mathrm{keV}$ and $4-11 \mathrm{keV}$, respectively. A hardness ratio (HR), shown in Fig. 7, is the ratio of the counting rate in the hard band over the soft band. There is a clear correlation of the HR with the rate, peaking when the rate is highest. The correlation factor between the rate and the HR is $r=0.76 \pm 0.12$. Even though the variability timescale here is much smaller, this behavior is compatible with the hardening reported in Chiappetti et al. (1999).

\subsection{EBL corrected spectrum}

For objects at non-negligible redshifts, the large, energydependent opacities can cause the emitted spectrum to be greatly modified both in shape and intensity (see e.g. Stecker et al. 1992; Biller 1995; Coppi \& Aharonian 1999; Vassiliev 2000). Unfortunately, at present the knowledge of the EBL still has large uncertainties, for both direct measurements and models, as summarized in Primack et al. (2001). In order to estimate the intrinsic $V H E$ spectrum, and thus to locate the Inverse Compton (IC) peak of the blazar's SED, we have used

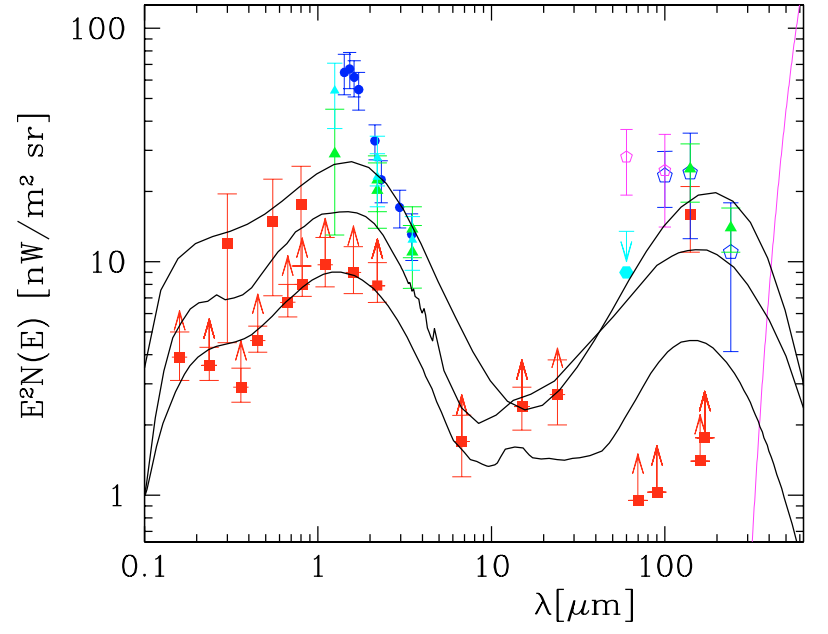

Fig. 8. Spectral energy distribution of the Extragalactic Background Light (EBL). It is thought to be characterized by two distinct bumps, around 1-2 and $100-200 \mu \mathrm{m}$, produced by the redshifted stellar light and re-radiated thermal dust emission, respectively. The compilation of measurements have been taken from Hauser \& Dwek (2001) and Aharonian et al. (2003). Lower limits are from HST and ISO source counts (Madau \& Pozzetti 2000; Elbaz et al. 2002; Gardner et al. 2000). Above $400 \mu \mathrm{m}$, the contribution of the CMB starts to dominate. The three continuous lines represent the "Primack-type" EBL shapes: from higher to lower fluxes (at $1 \mu \mathrm{m})$, Phigh, Primack01 and Primack04 models (see text). The steep line starting at $300 \mu \mathrm{m}$ is the onset of the CMBR.

three EBL models (Fig. 8) as representatives of three different flux levels for the stellar peak component. This is the EBL energy range which mostly affects the HESS spectrum: with data up to $3-3.5 \mathrm{TeV}$, the peak of the $\gamma-\gamma$ cross section is reached for soft photons with wavelengths $\lesssim 5 \mu \mathrm{m}$.

The three models used here are (in order from higher to lower fluxes): the phenomenological shape used in Aharonian et al. (2003), which is based on the original Primack et al. (2001) calculation but smoothed and scaled up to match the data points below $1 \mu \mathrm{m}$ and at $2-3.5 \mu \mathrm{m}$ (hereafter Phigh); the original Primack et al. (2001) calculation for a Salpeter initial mass function (hereafter Primack01); and the new 2004 calculation (Primack et al. 2004, hereafter Primack04), which takes advantage of the recent improvements in the knowledge of the cosmological parameters and of the local luminosity function of galaxies.

The opacities are calculated from the EBL SED shapes taking into account only the cosmology $\left(H_{0}=70 \mathrm{~km} \mathrm{~s}^{-1} \mathrm{Mpc}^{-1}\right.$, $\Omega_{\text {Mat }}=0.3$ and $\Omega_{\Lambda}=0.7$ ). To treat all three shapes similarly, no evolution has been introduced at this point. This corresponds to a "maximum absorption" hypothesis (i.e., for increasing $z$, constant instead of decreasing EBL comoving energy density). But at these redshifts $(\sim 0.1)$ and, for example, assuming the evolution given in Primack et al. (2001), the difference is still small (variation in the photon index $\Delta \Gamma<0.1$ in the range $0.3-1 \mathrm{TeV}$ ), and negligible compared to the differences between models.

The resulting absorption-corrected spectra are shown in Fig. 9, together with the observed spectrum. The intrinsic spectra are all well fitted by a single power-law model, with a hard 


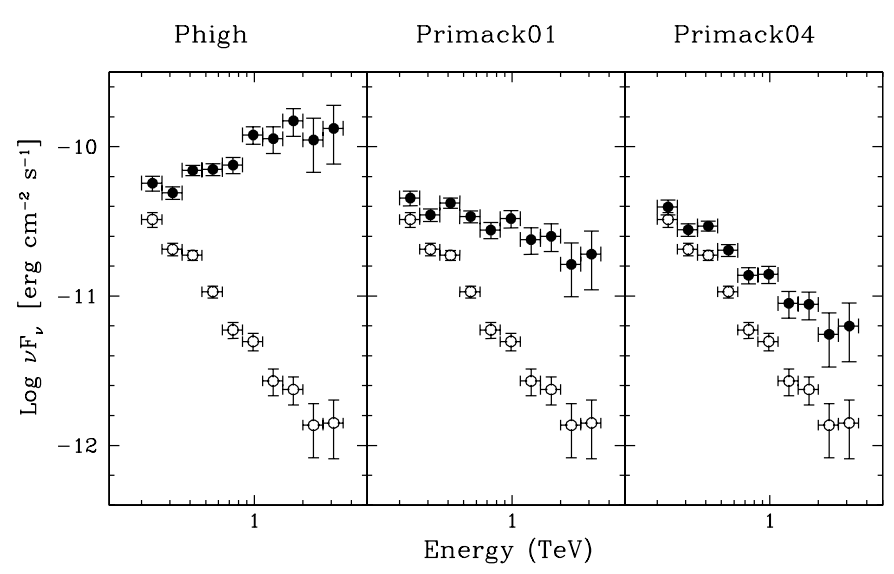

Fig. 9. PKS 2155-304 absorption-corrected spectra, along with the observed spectrum, for each EBL model considered and in a $v F_{v}$ plot (i.e. a zoom in the blazar's SED). Open dots: HESS observed spectrum. Filled dots: reconstructed intrinsic spectrum. All spectra from "Primack-type" models are compatible with a power-law shape, with different slopes: hard (Phigh) and soft (Primack01, Primack04), locating the intrinsic SED peak for the high component above $1-2 \mathrm{TeV}$ or below $200 \mathrm{GeV}$, respectively.

spectrum for Phigh $(\Gamma \approx 1.5)$, and soft spectra for Primack01 and Primack04 models $(\Gamma \approx 2.3$ and $\Gamma \approx 2.8$, respectively). This effect is directly related to the different flux levels of the stellar peak component, which imprint a different amount of softening onto the original spectrum. This direct link thus yields two simple scenarios for the location of the blazar's high energy peak, with the dividing line represented by the EBL flux which gives $\Gamma=2.0$ (1.3 times the Primack01 model). Models with stellar peak fluxes above this (such as Phigh, and generally all those in agreement with the direct estimates of the fluxes between 2 and $3.5 \mu \mathrm{m})$ imply a hard intrinsic spectrum $(\Gamma<2)$, and thus an IC peak above 1-2 TeV. EBL models with lower fluxes (such as the Primack01 and Primack04) imply instead a soft spectrum $(\Gamma>2)$, locating the IC peak below the observed energy range $(<200 \mathrm{GeV})$. In the following, we will discuss both scenarios for the SED modelling, using the Phigh and Primack2004 curves as the two ends of the possible range of values for the "Primack-type" shape (i.e., between the claimed EBL direct measurements at few microns and the lower limits from galaxy counts).

\section{SED modelling}

The broadband spectral morphology of PKS 2155-304 is typical of the BL Lac type, with a double-humped structure in $v F_{v}$ representation, exhibiting a low-energy and a high-energy component. Its broadband emission is usually attributed to emission from a beamed relativistic jet, oriented in a direction close to the line of sight (Begelman et al. 1984; Blandford \& Königl 1979). The spectral energy distribution in units of power per logarithmic bandwidth $E^{2} \mathrm{~d} N / \mathrm{d} E$ versus energy $E$ is shown in Fig. 10. The EGRET measurements, between the HESS and RXTE points, are from the third EGRET catalog (Hartman 1999) and from a very high $\gamma$-ray state described in Vestrand et al. (1995). There is a difference in spectral states, since in the former case the power law photon index is $1.71 \pm 0.24$ whereas it is $2.34 \pm 0.26$ in the latter which most likely consists of a mix of low and high activity state observations. The historical EGRET spectra are therefore unlikely to represent the state of PKS 2155-304 during the campaign presented here and are not used to put stringent constraints on the modelling. Considering the archival data and the steep X-ray spectrum in Fig. 10, the peak of the low-energy component occurs in the 2-2000 eV range. The archival BeppoSAX data from a high state analyzed by Chiappetti et al. (1999), and represented here above our RXTE data, show a peak at $\approx 0.1 \mathrm{keV}$. The absorbed VHE peak location is clearly below $300 \mathrm{GeV}$, with its exact location depending on the spectrum in the EGRET range.

Whereas the current models seem to agree that the lowenergy component is dominated by synchrotron radiation coming from nonthermal electrons emitted in collimated jets, the high-energy emission is assumed to be either inverse Compton scattering off the synchrotron photons (Synchrotron Self-Compton, SSC, see e.g. Maraschi et al. 1999; Bicknell \& Wagner 2002) or by external photons (see e.g. Sikora et al. 1994). This kind of leptonic model will be discussed in Sect. 4.2. A hadronic origin of the VHE emission using the Synchrotron-Proton Blazar (SPB) model with a dominating proton synchrotron component at high energies in a protonelectron plasma is also able to produce a double humped SED and is discussed in Sect. 4.3. The lack of correlation between the RXTE and HESS fluxes (and possibly also between the optical and the VHE emission) within the small variability range may indicate a different spatial origin, or a different underlying particle distribution. In the proton synchrotron model a lack of correlated variability between $\gamma$-ray emission and the low energy electron synchrotron component could arise if the electrons and protons are not co-accelerated.

The high-energy component above $\approx 100 \mathrm{GeV}$ is attenuated by interactions with the EBL and is a lower limit for the intrinsic spectrum. The energy budget in X-rays and VHE $\gamma$-rays is comparable, though the maximum output at the peak energy in the high-energy component is likely to be lower than that in the lower-energy component. Interpolating between the high-state EGRET archival data and VHE data would lead to a maximum located above $10 \mathrm{GeV}$, which is surprising since the observations reported here indicate a low state. Extrapolating the EGRET catalogue spectrum to VHE energies with a power law falls below the HESS data and therefore requires two inflexion points in the SED. Simultaneous observations in the $\mathrm{MeV}-\mathrm{GeV}$ range with the upcoming satellite GLAST will be crucial to constrain the high-energy component shape.

\subsection{Doppler boosting and synchrotron/Compton derived parameters}

The electromagnetic emission in blazars is very likely to be Doppler-boosted (or beamed) toward the observer. In the radio regime, the evidence for Doppler boosting in PKS 2155-304 comes from superluminal expansions observed with VLBI (Piner \& Edwards 2004). Relativistic beaming is also required in order to avoid absorption of $\mathrm{GeV}$ photons by 


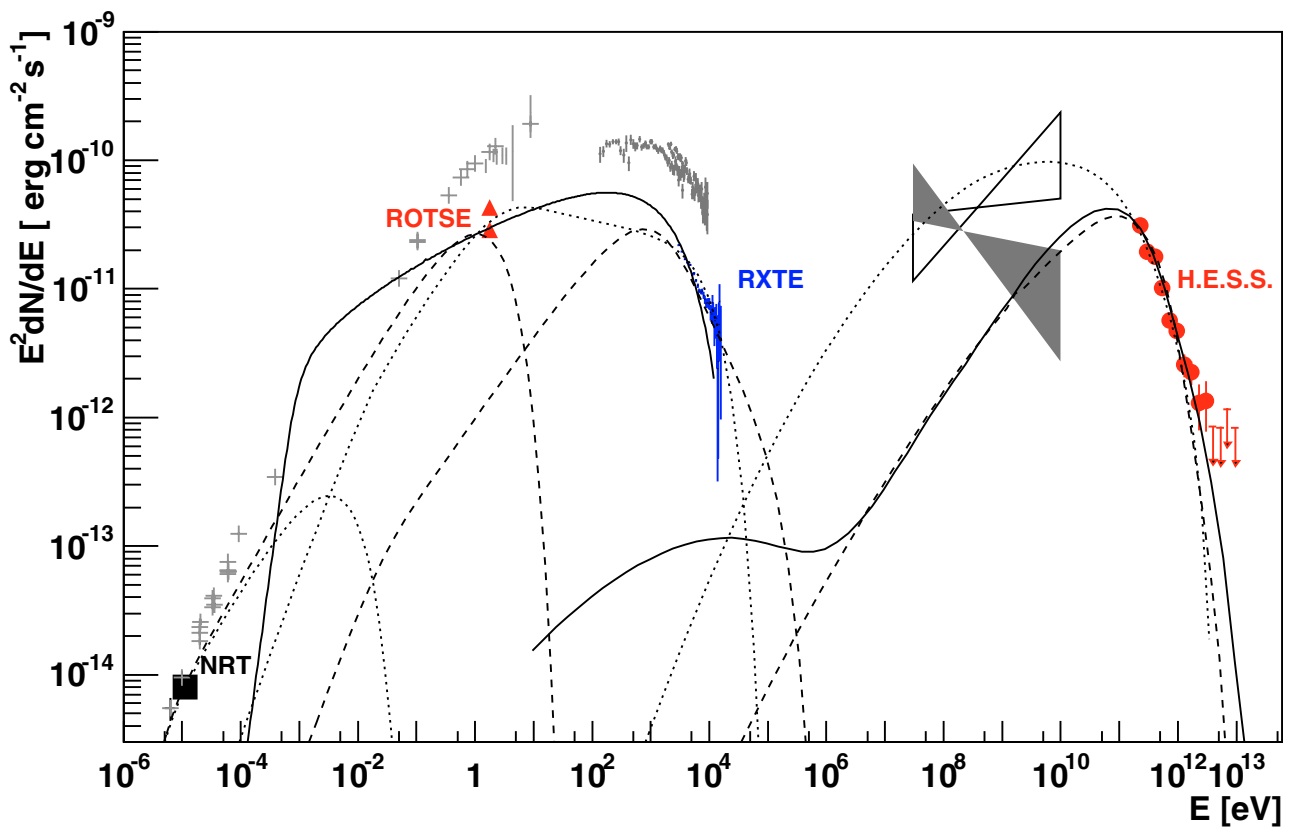

Fig. 10. Spectral energy distribution of PKS2155-304. Only simultaneous measurements are labeled. Non contemporaneous data are in grey symbols. The HESS spectrum is derived from October and November 2003 data (filled circles) as is the RXTE spectrum. The NRT radio point (filled square) is the average value for the observations carried out during this period. The two triangles are the highest and lowest ROTSE measurements for the Oct.-Nov. observations. Archival SAX data represent the high state observed in 1997 from Chiappetti et al. (1999). Archival EGRET data are from the third EGRET catalogue (shaded bowtie), Hartman (1999) and from a very high $\gamma$-ray state described in Vestrand et al. (1995) (open bowtie). 1995 UBVRI data are from Paltani et al. (1997) where the mean flux and flux deviation is used here. IUE data from Urry et al. (1993) at $2800 \AA$ and $1400 \AA$ are included here with an error bar corresponding to the spread of the measured fluxes. Other data are NED archival data. The solid line is the hadronic model described in Sect. 4.3. The dotted and dashed lines are the same leptonic model with different assumptions described in Sect. 4.2: the dotted line assumes a common origin for the optical and X-ray synchrotron emission, while the dashed line is the case where the optical emission emanates from the VLBI core; for both cases the lowest frequency hump is the predicted VLBI core emission. All the VHE emission in the models are absorbed according to the Primack04 model discussed in Sect. 3.4.

X-ray photons via the $\mathrm{e}^{+} / \mathrm{e}^{-}$pair-production process (see e.g. Maraschi et al. 1992). It is thus possible to use the $\gamma$-ray variability to establish a limit for the Doppler factor $\delta$, with $\delta$ defined in the standard way as $[\Gamma(1-\beta \cos \theta)]^{-1}$, where $\Gamma$ is the bulk Lorentz factor of the plasma in the jet, $\beta=v / c$, and $\theta$ is the angle to the line of sight.

Following Dondi \& Ghisellini (1995), the size of the $\gamma$-ray emission zone $R$ is derived from the time-scale of variability $t_{\mathrm{var}}$ (supposing the timescale of intrinsic variability is negligible compared to the light crossing time) by $R \leq c t_{\mathrm{var}} \delta /(1+z)$. In this case (assuming a time scale $t_{\mathrm{var}} \approx 2 \mathrm{ks}$ from Sect. 3 ) the size of the emission region is

$R \delta^{-1} \leq 5 \times 10^{13} \mathrm{~cm}$.

Since for $\gamma$-rays in the $300 \mathrm{GeV}-3 \mathrm{TeV}$ range the opacity $\tau_{\gamma \gamma}$ cannot significantly exceed 1 , independently of the emission mechanisms, another constraint on the minimum value of the Doppler factor can be derived by estimating how much Doppler boosting is necessary for photons with observed energy $E_{\gamma}$ to escape from a source with radius $R$ and a flux density $F\left(E_{t}\right)$, where $E_{t}=\left(m_{\mathrm{e}} c^{2}\right)^{2} \delta^{2} / E_{\gamma}(1+z)^{2}$. At this point one can follow Mattox et al. (1993) and especially Eq. (3.7) in Dondi \& Ghisellini (1995), writing that the opacity is

$\tau_{\gamma \gamma} \approx \frac{\sigma_{\mathrm{T}}}{5} \frac{1}{h c} \frac{d_{L}^{2}}{R} \frac{1}{\delta^{3}(1+z)} F\left(E_{t}\right)$.
Imposing that $\tau_{\gamma \gamma}<1$ for photons with observed energy $E_{\gamma}=$ $1 \mathrm{TeV}$ yields a lower limit on $\delta$ for given $R$ that can be derived numerically from the observed SED. For PKS 2155-304 this yields

$R^{-1} \delta^{-6.4} \leq 5.6 \times 10^{-24} \mathrm{~cm}^{-1}$.

The minimum allowable boost $\delta$ comes from combining Eqs. (2) and (4) and yields

$\delta \geq 19$

which is higher than the limit obtained in a similar way in Tavecchio et al. (1998), but at the lower end of the range usually obtained from SSC modelling as in Kataoka et al. (2000). It is useful to stress that this constraint is valid under the usual assumption that the region emitting the SED optical flux (i.e., the target photons) is cospatial with (or at least embeds) the high energy emitting region. The models used later in this paper are therefore essentially single zone models.

If the observed X-rays are synchrotron radiation from nonthermal electrons then the mean observed energy $\langle E\rangle$ of an electron with Lorentz factor $\gamma_{\mathrm{e}}$ is given by

$\langle E\rangle \approx \frac{\delta}{1+z} \frac{21 \hbar \gamma_{\mathrm{e}}^{2} q B}{15 \sqrt{3} m_{\mathrm{e}}}$.

Using $\langle E\rangle=10 \mathrm{keV}$ here yields

$B \delta \gamma_{\mathrm{e}}^{2}=1.1 \times 10^{12} \mathrm{G}$. 
In the SSC scenario the same electrons can Comptonize ambient photons up to the VHE regime, thus

$$
\frac{\delta}{1+z} \gamma_{\mathrm{e}} m_{\mathrm{e}} c^{2} \geq 3 \mathrm{TeV} \text {. }
$$

Combining Eqs. (6) and (7) yields

$B \delta^{-1} \leq 1.1 \times 10^{12} \mathrm{G} \times\left(\frac{3 \mathrm{TeV}}{m_{\mathrm{e}} c^{2}}\right)^{-2}(1+z)^{-2}$.

A numerical application yields

$B \delta^{-1} \leq 0.03 \mathrm{G}$

and hence the Lorentz factor is constrained to $\gamma_{\mathrm{e}} \geq 1.3 \times 10^{6}$ which is higher than that calculated as above for Mkn 421 by Takahashi et al. (1996) who derived $\gamma_{\mathrm{e}}>5 \times 10^{5}$ (and $B \approx 0.2 \mathrm{G}$ ) but lower than $\gamma_{\mathrm{e}} \approx 10^{7}$ found in a similar way in 1ES1959+650 (Giebels et al. 2002).

The X-ray data presented above imply that the X-ray spectrum of PKS 2155-304 hardens as the source brightens. This is often measured in BL Lac objects; a hardening of the spectrum when flares occur, and a blueward shift of the peak of the synchrotron emission $v_{\text {sync }}$ (and presumably higher energy inverse-Compton emission) by factors that can be as large as 100 were measured in the cases of Mkn 501 (Pian et al. 1998), 1ES 1426+428 and PKS 0548-322 (Costamante et al. 2001). In the case of PKS 2005-489 (Perlman et al. 1999), a more moderate shift of a factor of 3 or less of the synchrotron emission was found. The archival data suggest that $v_{\text {sync }}$ lies in the UV band for PKS 2155-304, but no data were taken simultaneously in this campaign at that wavelength.

The lack of indication for correlated X-ray/VHE variability does not imply that PKS 2155-304 behaves differently from VHE sources such as Mkn 421, for which VHE/X-ray correlation has been established on a much higher variability basis (see e.g. Cui et al. 2004) with dynamical ranges of 30 in both energy bands. Limiting those observations to the same dynamical range as observed here would not allow any claim for correlation. Future observations of PKS 2155-304 with a higher variability amplitude would bring more insight into this.

\subsection{Leptonic interpretation}

Interpretation with a single zone SSC model of the SED of PKS 2155-304 has already been proposed in the literature using two different assumptions. In Kataoka et al. (2000) the low energy tail of the SSC model is used to account for the low-energy component up to the optical in the SED. That component is decomposed into two sub-components by Chiappetti et al. (1999) where the radio to optical emission has another origin than the X-rays, which are assumed to come from the jet. These two different interpretations are used here in the context of the leptonic model described in Katarzyński et al. (2001) which has already been applied to Mkn 501 and Mkn 421 (Katarzyński et al. 2003). To constrain this model, only the simultaneous data are used, since the archival data reported in the SED of Fig. 10 are likely to not represent the state of this source (note for example the difference in optical flux and the ROTSE measurement).
When using the Primack04 absorption, the model used here can reproduce the X-ray through VHE part of the SED, but the HESS spectrum constrains it such that the radio measurement can not be included in the synchrotron bump predicted by the single-zone model. As for Mkn 421 and Mkn 501, adding a more extended component than the VHE emitting zone can provide an explanation for this. The origin is probably the compact VLBI core which has a radio core to lobe ratio of $\approx 1$ (Laurent-Muehleisen et al. 1993; Piner \& Edwards 2004) and a typical size of $10^{18} \mathrm{~cm}$, more than two orders of magnitude larger than the VHE emitting zone. This VLBI feature dominates the spectrum at low energy and is included in the SED modelling here. An uncertainty remains which is the high frequency cutoff of this VLBI component. The host galaxy contribution to the optical flux is estimated to be $\approx 10^{-11} \mathrm{erg} \mathrm{cm}^{-2} \mathrm{~s}^{-1}$, deduced from the magnitudes given by KFS 98 and assuming a low-redshift solar metallicity elliptical galaxy of age equal to $13 \mathrm{Gyr}(R-H=2.4)$, corresponding to a mass of $5 \times 10^{11} M_{\odot}$ (Fioc \& Rocca-Volmerange 1997). So even at the measured low activity state of PKS 2155-304 the host galaxy is not contributing much in the optical range.

The ROTSE measurement can be ascribed here to either the high-energy tail of the VLBI component or to the synchrotron part of the SSC model. Assuming a common origin for the $\mathrm{X}$-rays and the optical emission, and using a variability time scale of $0.1 \mathrm{~d}$ to constrain the emitting zone, the model tends to predict a high IC flux, as shown in Fig. 10. However, the lack of correlation between the X-rays and the optical emission in our measurements - also suggested by Dominici et al. (2004) based on less sensitive RXTE/ASM measurements - indicates that the optical emission could originate from the VLBI component, which is modelled by a slight increase in the maximal Lorentz factor of the emitting electrons. This in turn lessens the constraint on the simultaneous SSC fit of the X-ray and VHE part and allows a better fit of the VHE spectrum for smaller sizes of the emitting zone. Detailed parameters of the two hypotheses are given in Table 1.

If the absorption correction is well described by the Phigh model, the slope of HESS data at high energy implies that the peak of the $\mathrm{TeV}$ emission bump is located above $4 \mathrm{TeV}$ (or $10^{27} \mathrm{~Hz}$ ). Such a high frequency peak emission imposes a strong constraint for the single-zone SSC scenario, especially since the peak of the synchrotron bump has to remain below $1 \mathrm{keV}$ (or $10^{17.5} \mathrm{~Hz}$ ) as required by the slope of the RXTE data. High values of both the jet Doppler factor and the maximal Lorentz factor of radiating particles are required to reach the necessary energy for the IC bump, that is $\gamma_{\max } \delta>8 \times 10^{6}$. On the other hand, to keep the synchrotron peak below $1 \mathrm{keV}$ imposes an upper limit to the magnetic field. Within these constrains, the best fit we obtain is shown in Fig. 11. We can note that none of the high energy tails are well accounted for. The set of parameters for the best fit is given in Table 1. This fit marginally reproduces the observed X-ray and $\gamma$-ray data, but is not as satisfactory than that obtained with the Primack04 absorption correction, and in any case it is impossible to take into account the ROTSE optical point. The main changes in parameters between the two fits consist in enhancing the boosting, 
Table 1. Parameters for the SED fit with the assumption that the optical and X-ray emission are part of the jet synchrotron emission (Model 1 in the table, dotted line in Fig. 10) or that the optical emission emanates from the VLBI emission zone (Model 2 in the table, dashed lines in the same figure). The parameters are described in Katarzyński et al. (2001).

\begin{tabular}{cccc}
\hline \hline Parameter & Model 1 & Model 2 & Model 2 Phigh \\
\hline$R_{\text {blob }}\left(10^{15} \mathrm{~cm}\right)$ & 6.5 & 1.5 & 1.5 \\
$B(\mathrm{G})$ & 0.15 & 0.25 & 0.02 \\
$\delta_{\mathrm{b}}$ & 25 & 25 & 50 \\
$K\left(\mathrm{~cm}^{-3}\right)$ & 160 & $2.0 \times 10^{3}$ & 240 \\
$\gamma_{\text {break }}\left(10^{3}\right)$ & 7.5 & 100 & 300 \\
$\gamma_{\text {max }}\left(10^{5}\right)$ & 3.8 & 9.0 & 5.0 \\
$\alpha_{1}$ & 1.4 & 1.7 & 1.6 \\
$\alpha_{2}$ & 3.2 & 4.65 & 4.5 \\
$R_{\text {jet }}\left(10^{6} \mathrm{~cm}\right)$ & 1.0 & 1.0 & 1.0 \\
$L_{\text {jet }}(\mathrm{pc})$ & 55 & 55 & 55 \\
$\delta_{\text {jet }}$ & 2 & 2 & 2 \\
$K_{\text {jet }}\left(\mathrm{cm}^{-3}\right)$ & 40 & 40 & 40 \\
$B_{\text {jet }}(\mathrm{G})$ & 0.04 & 0.04 & 0.04 \\
$\gamma_{\text {break,jet }}\left(10^{3}\right)$ & 2.5 & 45 & 45 \\
\hline
\end{tabular}

which then becomes quite extreme, while reducing the density and magnetic field for the Phigh absorption correction.

The constraints on $\delta$ derived here from either simple opacity arguments or from the one-zone model parametrisation of the SED are in the range of Doppler factors usually derived with such assumptions or models for other VHE emitters. As pointed out by Chiaberge et al. (2000) such high values are however at odds with attempts to unify the BL Lac population with the family of FR I sources (Urry \& Padovani 1995), the latter being possibly an unbeamed since off-axis viewed case of the former. The same authors suggest that models where velocity structures in the jet, such as the "spine-sheath" model (see e.g. Sol et al. 1989) or the decelerating flow model (Georganopoulos \& Kazanas 2003; Georganopoulos \& Kazanas 2004) allow lower bulk Lorentz factors. Another option from Pelletier et al. (2004) is to cope with the pair creation catastrophe implied by smaller Doppler factors in their "two-flow" solution. Comparing the SED with such models, which make the BL Lac - FR-I connection more plausible, is an interesting task but beyond the scope of this paper.

\subsection{Hadronic models}

Generally, the leptonic models constitute the preferred concept for TeV blazars, essentially because of two attractive features: (i) the capability of the (relatively) well understood shocks to accelerate electrons to TeV energies (Sikora \& Madejski 2001; Pelletier 2001) and (ii) the effective conversion of the kinetic energy of these relativistic electrons into the X-ray and VHE $\gamma$-ray emission components through the synchrotron and inverse Compton radiation channels. The hadronic models are generally lack these virtues. They assume that the observed $\gamma$-ray emission is initiated by accelerated protons interacting

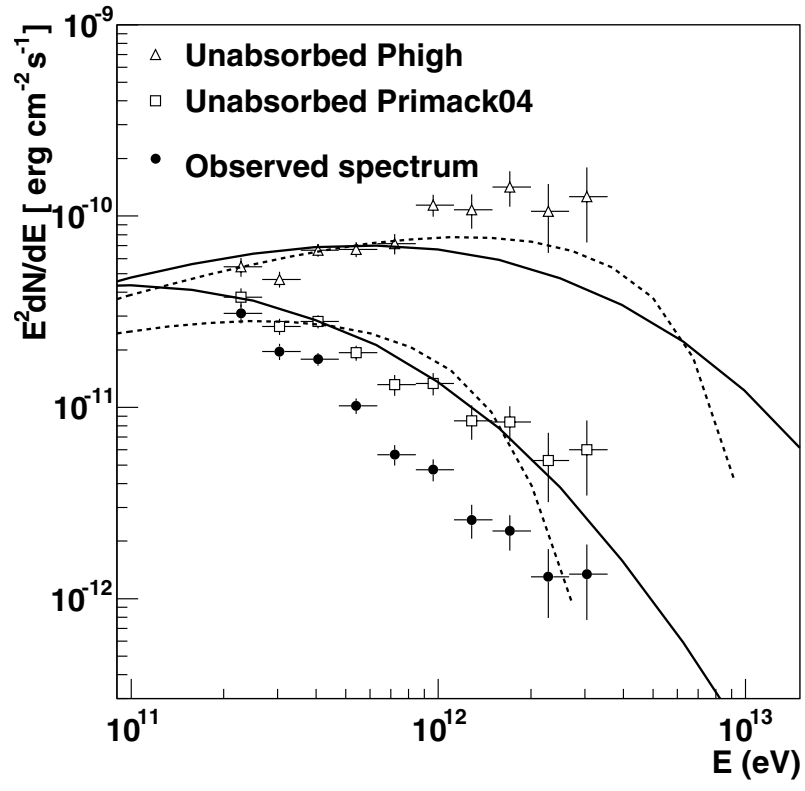

Fig. 11. Estimations of the intrinsic PKS 2155-304 VHE spectrum, corrected for either the Phigh or Primack04 models, along with the associated intrinsic (i.e. before absorption) SSC model (dashed lines) and SPB model (solid lines).

with ambient matter (Bednarek 1993; Dar \& Laor 1997; Pohl \& Schlickeiser 2000), photon fields (PIC model, Mannheim 2000), magnetic fields (Aharonian et al. 2002) or both (Mücke \& Protheroe 2000).

The models of $\mathrm{TeV}$ blazars involving interactions of protons with photon and $\mathrm{B}$-fields require particle acceleration to extreme energies exceeding $10^{19} \mathrm{eV}$ which is possible if the acceleration time is close to $t_{\mathrm{acc}}=r_{\mathrm{g}} / c$ ( $r_{\mathrm{g}}$ is the gyro-radius). This corresponds (independent of a specific acceleration mechanism) to the maximum (theoretically possible) acceleration rates (Aharonian et al. 2002) which can only be achieved by the conventional diffusive shock acceleration in the Bohm diffusion regime.

On the other hand, the condition of high efficiency of radiative cooling of accelerated particles requires extreme parameters characterizing the sub-parsec jets and their environments, in particular very high densities of the thermal plasma, radiation and/or B-fields. In particular, the proton-synchrotron models of $\mathrm{TeV}$ blazars require highly magnetized $(B \gg 10 \mathrm{G})$ condensations of $\gamma$-ray emitting clouds containing Extremely High Energy (EHE) protons, where the magnetic pressure dominates over the pressure of relativistic protons (Aharonian 2000).

Below we use the hadronic SPB model (Mücke \& Protheroe 2000, 2001) to model the average spectral energy distribution (SED) of PKS 2155-304 in October-November 2003. A detailed description of the model, and its implementation as a (time-independent) MonteCarlo/numerical code, has been given in Mücke et al. (2003) and Reimer et al. (2004).

Considering the rather quiet activity state of PKS 2155-304 in Oct.-Nov. 2003, we use the 3EG catalog spectrum, since it is the best determined EGRET spectrum 
from this source to date, as an upper limit for modelling purposes.

Flux variability provides an upper limit for the size of the emission region. To allow for a comparative study between leptonic and hadronic models we fix here the comoving emission region to $R \sim c t_{\mathrm{var}} \delta=5 \times 10^{13} \delta \mathrm{cm}$ deduced from the X-ray variability. We assume that the optical through $\mathrm{X}$-ray emission and the $\gamma$-ray output stem from the same region of size $R$.

A reasonable model representation for the simultaneous data assuming a Primack04 model for the VHE absorption is found for the following parameters: magnetic field $B=40 \mathrm{G}$, Doppler factor $\delta=20$, injection electron spectral index $\alpha_{\mathrm{e}}=$ 1.6, assumed to be identical to the injection proton spectral index $\alpha_{\mathrm{p}}$, maximum proton energy of order $\gamma_{\mathrm{p}, \max } \sim 4 \times 10^{9}$, e/p-ratio of 0.15 and a near-equipartition proton energy density of $u_{\mathrm{p}}=27 \mathrm{erg} \mathrm{cm}^{-3}$. The required total jet power is of the order $L_{\text {jet }} \sim 1.6 \times 10^{45} \mathrm{erg} \mathrm{s}^{-1}$. When using the Phigh EBL model, a reasonable representation of the data may be achieved by increasing the maximum injected proton energy to $\gamma_{\mathrm{p}, \max }=10^{10}$ and simultaneously increasing the e/p-ratio to 0.24 , while all other parameters remain unchanged. Note that here the maximum proton gyro-radius approaches the size of the emission region. Alternatively, a doubling of the magnetic field to $80 \mathrm{G}$ together with an increase of $\gamma_{\mathrm{p}, \max }$ to $8 \times 10^{9}$ and a e/p-ratio of unity (leading to $u_{B} \approx 50 u_{\mathrm{p}}$ ) represents the SED-data equally well. In conclusion, none of the "Primacktype" EBL models can explicitly be ruled out in the framework of the SPB-model by the HESS data presented here. In all cases, proton synchrotron emission dominates the (sub-) TeV radiative output. Depending on the Doppler factor, part of the proton synchrotron radiation produced may be reprocessed to lower energies. Contributions from the muon and pion cascades are always lower than the proton synchrotron component. The low energy component is dominated by synchrotron radiation from the primary electrons, with a negligible contribution of synchrotron radiation from secondary electrons (produced by the p- and $\mu^{ \pm}$-synchrotron cascade).

On the other hand, the synchrotron radiation of secondary electrons resulting from interactions of VHE $\gamma$-rays with external low-energy photons with a modest $\gamma \gamma \rightarrow \mathrm{e}^{+} / \mathrm{e}^{-}$opacity $\left(\tau_{\gamma \gamma} \leq 1\right)$ may lead to significant X-ray emission with a luminosity comparable to the luminosity of the primary VHE emission (Aharonian 2000).

Models involving meson production inevitably predict neutrino emission due to the decay of charged mesons. The SPB-model for PKS 2155-304 explains the high energy emission dominantly as proton synchrotron radiation, making the neutrino flux completely negligible.

\section{Conclusions}

This paper reports multi-wavelength observations of the BL Lac object PKS 2155-304 in 2003. Although the source appeared variable in the VHE, X-rays and optical bands, the latter two indicate that PKS 2155-304 was in a state close to historically low measurements, even though it was easily detectable by HESS in all nights of observations since the beginning of the detector operation (see AH04 for the observation history up to August 2003). An extreme case of VHE variability shows a peak-to-peak increase of a factor of $2.5 \pm 0.9$ in $0.09 \mathrm{~d}$. Variability on the timescale of a few ks in the 2$10 \mathrm{keV}$ band and of the order of $0.1 \mathrm{~d}$ for energies $>300 \mathrm{GeV}$ were observed by RXTE and HESS The X-ray data show a correlation of the flux with the spectrum, which becomes harder when the source is brighter. At the level of the simultaneously observed modest variability, no correlation between the VHE $\gamma$-rays, the X-rays and optical was seen. Observations with greater variability and better coverage are needed before it can be asserted that the VHE/X-ray pattern in PKS 2155-304 is different from other known VHE-emitting AGN. Since the source was in a low emission state in both the optical and Xrays compared to archival measurements, this lack of correlation has yet to be established for a higher emission state. Simultaneous observations in the X-rays/optical band and VHE $\gamma$-rays had never previously been performed on this scale. Its continual VHE detection makes PKS 2155-304 unique in the TeV BL Lac category, and probably indicates that HESS has achieved the sensitivity level where it can detect the quiescent state of PKS 2155-304 at any time. A time-averaged energy spectrum is determined for the 2 observation periods and fits to a power law $(\Gamma=3.37 \pm 0.07)$ in the VHE $\gamma$-rays, and to a broken power law $\left(\Gamma_{L}=2.81 \pm 0.05, E_{\mathrm{b}}=4.9 \pm 0.8 \mathrm{keV}\right.$, $\left.\Gamma_{\mathrm{H}}=2.95 \pm 0.04\right)$ in the X-rays.

A comparison of the intrinsic spectrum with predictions from existing one-zone leptonic and one-zone hadronic models is attempted to give a plausible estimation of underlying physical parameters. The values of the parameters are in line with those inferred for other VHE-emitting blazars. In these models the VHE emission is attenuated according to two different EBL levels. This changes mainly the Doppler boosting in the leptonic model, but the high level EBL decreases the agreement significantly. In the hadronic model, the maximum injected proton energy can be changed to accomodate different EBL levels and can therefore not significantly constrain any of the EBL models used here.

Acknowledgements. The authors would like to thank the anonymous referee for his correction and useful comments that improved this paper. The support of the Namibian authorities and of the University of Namibia in facilitating the construction and operation of HESS is gratefully acknowledged, as is the support by the German Ministry for Education and Research (BMBF), the Max-Planck-Society, the French Ministry for Research, the CNRS-IN2P3 and the Astroparticle Interdisciplinary Programme of the CNRS, the UK Particle Physics and Astronomy Research Council (PPARC), the IPNP of the Charles University, the South African Department of Science and Technology and National Research Foundation, and by the University of Namibia. We appreciate the excellent work of the technical support staff in Berlin, Durham, Hamburg, Heidelberg, Palaiseau, Paris, Saclay, and in Namibia in the construction and operation of the equipment.

The authors acknowledge the support of the ROTSE III collaboration and the sharing of observation time with the Australian ROTSE IIIa telescope operated by A. Phillips and M. C. B. Ashley from the School of Physics, Department of Astrophysics and Optics, University of New South Wales, Sydney, Australia. Special thanks also to R. Quimby from the University of Texas for providing tools for data-reduction. H. Sol and C. Boisson thank K. Katarzyński for his SSC code. This research has made use of the NASA/IPAC Extragalactic Database 
(NED) which is operated by the Jet Propulsion Laboratory, California Institute of Technology, under contract with the National Aeronautics and Space Administration. We thank the RXTE team for their prompt response to our $\mathrm{ToO}$ request and the professional interactions that followed.

\section{References}

Aharonian, F. 2000, New Astron., 5, 377

Aharonian, F., Belyanin, A. A., Derishev, E. V., \& Kocharovsky, V. V. 2002, Phys. Rev. D, 66, 023005

Aharonian, F. A., Akhperjanian, A., Beilicke, M., et al. 2003, A\&A, 403,523

Aharonian, F. A., Akhperjanian, A. G., Aye, K.-M., et al. 2004, APh, 22, 109

Aharonian, F. A., et al. 2005, A\&A, 430, 865

Akerlof, C. W., Balsano, R., Barthelmy, S., et al. 2000, ApJ, 542, 251

Akerlof, C. W., Kehoe, R. L., McKay, T. A., et al. 2003, PASP, 115, 132

Bednarek, W. 1993, ApJ, 402, L29

Begelman, M. C., Blandford, R. D., \& Rees, M. J. 1984, Rev. Mod. Phys., 56, 255

Bernlöhr, K., Carrol, O., Cornils, R., et al. 2003, APh, 20, 111

Bicknell, G. V., \& Wagner, S. J. 2002, PASA, 19, 129

Biller, S. D. 1995, APh, 3, 385

Blandford, R. D., \& Königl, A. 1979, ApJ, 232, 34

Catanese, M., \& Sambruna, R. M. 2000, ApJ, 534, L39

Chadwick, P. M., Lyons, K., McComb, T. J. L., et al. 1999, ApJ, 513, 161

Chiaberge, M., Celotti, A., Capetti, A., \& Ghisellini, G. 2000, A\&A, 358,104

Chiappetti, L., Maraschi, L., Tavecchio, F., et al. 1999, ApJ, 521, 522

Coppi, P. S., \& Aharonian, F. A. 1999, APh, 11, 35

Cornils, R., Gillessen, S., Jung, I., et al. 2003, APh, 20, 129

Costamante, L., Ghisellini, G., Tagliaferri, G., et al. 2001, A\&A, 371, 512

Costamante, L., Aharonian, F., Horns, D., \& Ghisellini, G. 2004, NewAR, 48, 469

Cui, W., et al. 2004, AIP Conf. Proc. [arXiv: astro-ph/0410160]

Dominici, T. P., Abraham, Z., Teixeira, R., \& Benevides-Soares, P. 2004, AJ, 128, 47

Dondi, L., \& Ghisellini, G. 1995, MNRAS, 273, 583

Elbaz, D., Cesarsky, C. J., Chanial, P., et al. 2002, A\&A, 384, 848

Falomo, R., Bersanelli, M., Bouchet, P., \& Tanzi, E. G. 1993, AJ, 106, 11

Falomo, R. 1996, MNRAS, 283, 241

Fioc, M., \& Rocca-Volmerange, B. 1997, A\&A, 326, 950

Funk, S., Hermann, G., Hinton, J., et al. 2004, APh, 22, 285

Gardner, J. P., Brown, T. M., Ferguson, H. C., et al. 2000, ApJ, 542, L79

Gaidos, J. A., Akerlof, C. W., Biller, S. D., et al. 1996, Nature, 383, 319

Georganopoulos, M., \& Kazanas, D. 2003, ApJ, 594, L27

Georganopoulos, M., \& Kazanas, D. 2004, NewAR, 48, 403

Giebels, B., Bloom, E. D., Focke, W., et al. 2002, ApJ, 571, 763

Giommi, P., Fiore, F., Guainazzi, M., et al. 1998, A\&A, 333, L5

Hartman, R. C., Bertsch, D. L., Bloom, S. D., et al. 1999, ApJS, 123, 79

Hauser, M. G., \& Dwek, E. 2001, ARA\&A, 39
Hofmann, W. 2002, HESS project in The Universe Viewed in Gamma-rays, September 25, Kashiwa, Japan

Jahoda, K., et al. 1996, in EUV, X-ray, and Gamma-ray Instrumentation for Astronomy VII, SPIE Proc., 2808, 59

Kataoka, J., Takahashi, T., Makino, F., Inoue, S., et al. 2000, ApJ, 528, 243

Katarzyński, K., Sol, H., \& Kus., A. 2001, A\&A, 367, 809

Katarzyński, K., Sol, H., \& Kus., A. 2003, A\&A, 410, 101

Kirk, J. G., \& Mastichiadis, A. 1999, APh, 11, 45

Kotilainen, J. K., Falomo, R., \& Scarpa, R. 1998, A\&A, 336, 479

Dar, A., \& Laor, A. 1997, ApJ, 478, L5

Laurent-Muehleisen, S. A., Kollgaard, R. I., Moellenbrock, G. A., \& Feigelson, E. D. 1993, AJ, 106, 875

Madau, P., \& Pozzetti, L. 2000, MNRAS, 312, L9

Mannheim, K. 2000, A\&A, 269, 67

Madejski, G., Takahashi, T., Tashiro, M., et al. 1996, ApJ, 459, 156

Maraschi, L., Ghisellini, G., \& Celotti, A. 1992, ApJ, 397, L5

Maraschi, L., Fossati, G., Tavecchio, F., et al. 1999, ApJ, 526, L81

Marshall, H. L., Fruscione, A., \& Carone, T. E. 1995, ApJ, 439, 90

Mattox, J. R., Bertsch, D. L., Chiang, J., et al. 1993, ApJ, 410, 609

Mücke, A., \& Protheroe, R. J. 2000, AIP Conf. Proc., 515, 149

Mücke, A., \& Protheroe, R. J. 2001, APh, 15, 121

Mücke, A., Protheroe, R. J., Protheroe, R. J., et al. 2003, APh, 18, 593

Paltani, S., Courvoisier, T. J.-L., Protheroe, R. J., Blecha, A., \& Bratschi, P. 1997, A\&A, 327, 539

Pelletier, G. 2001, AIP Conf. Proc., 558, 289

Pelletier, G., et al. 2004, AIP Conf. Proc., 745, 34

Perlman, E. S., Madejski, G., Stocke, J. T., \& Rector, T. A. 1999, ApJ, 523, L11

Pian, E., Vacanti, G., Tagliaferri, G., et al. 1998, ApJ, 492, L17

Piner, G. P., \& Edwards, P. G. 2004, ApJ, 600, 115

Pohl, M., \& Schlickeiser, R. 2000, A\&A, 354, 395

Primack, J., et al. 2001, AIP Conf. Proc., 558, 463

Primack, J., Bullock, J., \& Somerville, R. 2004, Observational Gamma-Ray Cosmology, Proc. of the Conf. Gamma 2004 Heidelberg, 26-30 July, Germany

Reimer, A., Protheroe, R. J., \& Donea, A.-C. 2004, A\&A, 419, 89

Rybicki, G. B., \& Lightman, A. P. 1979, Radiative Processes in Astrophysics (New York: Wiley)

Sembay, S., Warwick, R. S., Urry, C. M., et al. 1993, ApJ, 404, 112

Sikora, M., Begelman, M. C., \& Rees, M. J. 1994, ApJ, 421,153

Sol, H., Pelletier, G., \& Asseo, E. 1989, MNRAS, 237, 411

Stecker, F. W., de Jager, O. C., \& Salamon, M. H., et al. 1992, ApJ, 390, L49

Sikora, M., \& Madejski, G. 2001, AIP Conf. Proc., 558, 275

Takahashi, T., Tashiro, M., Madejski, G., et al. 1996, ApJ, 470, L89

Tavecchio, F., et al. 1998, A\&A, 509, 608

Treves, A., Morini, M., Chiappetti, L., et al. 1989, ApJ, 341, 733

Urry, C. M., \& Padovani, P. 1995, PASP, 107, 803

Urry, C. M., Maraschi, L., Edelson, R., et al. 1993, ApJ, 411, 614

Urry, C. M., et al. 1997, ApJ, 486, 770

van Driel, W., Pezzani, J., \& Gérard, E. 1996, in High sensitivity radio astronomy, ed. N. Jackson, \& R. J. Davis (Cambridge Univ. Press), 229

Vassiliev, S. D. 2000, APh, 12, 217

Vestrand, W. T., Stacy, J. G., \& Sreekumar, P. 1995, ApJ, 454, L93

Vestrand, W. T., \& Sreekumar, P. 1999, APh, 11, 197

Xue, Y., \& Cui, W. 2004, ApJ, in press [arXiv: astro-ph/0412226]

Zhang, Y. H., Treves, A., et al. 2002, ApJ, 572, 762 